\title{
Lymphatic vessels in human adipose tissue
}

\author{
Patricia de Albuquerque Garcia Redondo ${ }^{1,2}$ - Fernanda Gubert ${ }^{3,4}$. Camila Zaverucha-do-Valle ${ }^{4,5}$. \\ Tatiana Pereira Pena Dutra ${ }^{6}$. Jackline de Paula Ayres-Silva ${ }^{7} \cdot$ Natasha Fernandes ${ }^{8}$. \\ Antonio Augusto Peixoto de Souza ${ }^{9} \cdot$ Marilena Loizidou $^{2} \cdot$ Christina Maeda Takiya $^{8}$. \\ Maria Isabel Doria Rossi ${ }^{1,10} \cdot$ Radovan Borojevic $^{11}$
}

Received: 1 November 2018 / Accepted: 19 September 2019 / Published online: 27 November 2019

(C) Springer-Verlag GmbH Germany, part of Springer Nature 2019

\begin{abstract}
Despite being considered present in most vascularised tissues, lymphatic vessels have not been properly shown in human adipose tissue (AT). Our goal in this study is to investigate an unanswered question in AT biology, regarding lymphatic network presence in tissue parenchyma. Using human subcutaneous (S-) and visceral (V-) AT samples with whole mount staining for lymphatic specific markers and three-dimensional imaging, we showed lymphatic capillaries and larger lymphatic vessels in the human VAT. Conversely, in the human SAT, microcirculatory lymphatic vascular structures were rarely detected and no initial lymphatics were found.
\end{abstract}

Keywords Lymphatics $\cdot$ Visceral $\cdot$ Subcutaneous $\cdot$ Adipose $\cdot$ Vessels

\section{Introduction}

The lymphatic system, an essential component of the immunological system, is responsible for draining the remaining $10 \%$ of interstitial fluid that does not return to blood veins through an extensive network of capillaries and vessels spread throughout the body (Jeltsch et al. 2003; Tammela and Alitalo 2010). Lymphatics are connected to lipid metabolism by lymphatic vessels in the small intestine and mesentery, which transport vitamins and lipids from diet (Kohan et al. 2011; Tammela and Alitalo 2010), as well as by the involvement in the removal

Patricia de Albuquerque Garcia Redondo prbioeng@gmail.com

1 Programa de Ciências Morfológicas, Instituto de Ciências Biomédicas, Universidade Federal do Rio de Janeiro, Rio de Janeiro, RJ, Brazil

2 Research Department of Surgical Biotechnology, Division of Surgery and Interventional Science, University College London, London, UK

3 Instituto de Ciências Biomédicas, Universidade Federal do Rio de Janeiro, Rio de Janeiro, RJ, Brazil

4 Laboratório de Neurobiologia Celular e Molecular, Instituto de Biofísica Carlos Chagas Filho, Universidade Federal do Rio de Janeiro, Rio de Janeiro, RJ, Brazil

5 Instituto Nacional de Infectologia Evandro Chagas, Fundação Oswaldo Cruz, Rio de Janeiro, RJ, Brazil of cholesterol from peripheral tissues (Nanjee et al. 2001; Martel et al. 2013; Randolph and Miller 2014). In spite of these associations and of being considered present in virtually all vascularised tissues of the human body, with the exception of bone (Tammela and Alitalo 2010), initial lymphatic vessels have been rarely described in adipose tissue. Previous studies have shown adipose tissue associated with collecting lymphatic vessels in lymph nodes (Wang and Oliver 2010), in ectopic deposits such as the epicardial adipose tissue (Montani et al. 2004) and in fat deposits adjacent to the aorta (Martel et al. 2013). In the few available descriptions, lymphatic capillaries were not found in

6 Instituto de Bioquímica Médica Leopoldo de Meis, Universidade Federal do Rio de Janeiro, Rio de Janeiro, RJ, Brazil

7 Laboratório de Patologia, Fundação Oswaldo Cruz, Rio de Janeiro, RJ, Brazil

8 Laboratório de Patologia Celular, Instituto de Biofísica Carlos Chagas Filho, Universidade Federal do Rio de Janeiro, Rio de Janeiro, RJ, Brazil

9 Departamento de Cirurgia, Faculdade de Medicina, Universidade Federal do Rio de Janeiro, Rio de Janeiro, RJ, Brazil

10 Programa de Ciências Morfológicas, Instituto de Ciências Biomédica, Hospital Universitário Clementino Fraga Filho, Universidade Federal do Rio de Janeiro Rio de Janeiro Brazil, Rio de Janeiro, RJ, Brazil

11 Centro de Medicina Regenerativa, Faculdade de Medicina de Petrópolis, Petrópolis, RJ, Brazil 

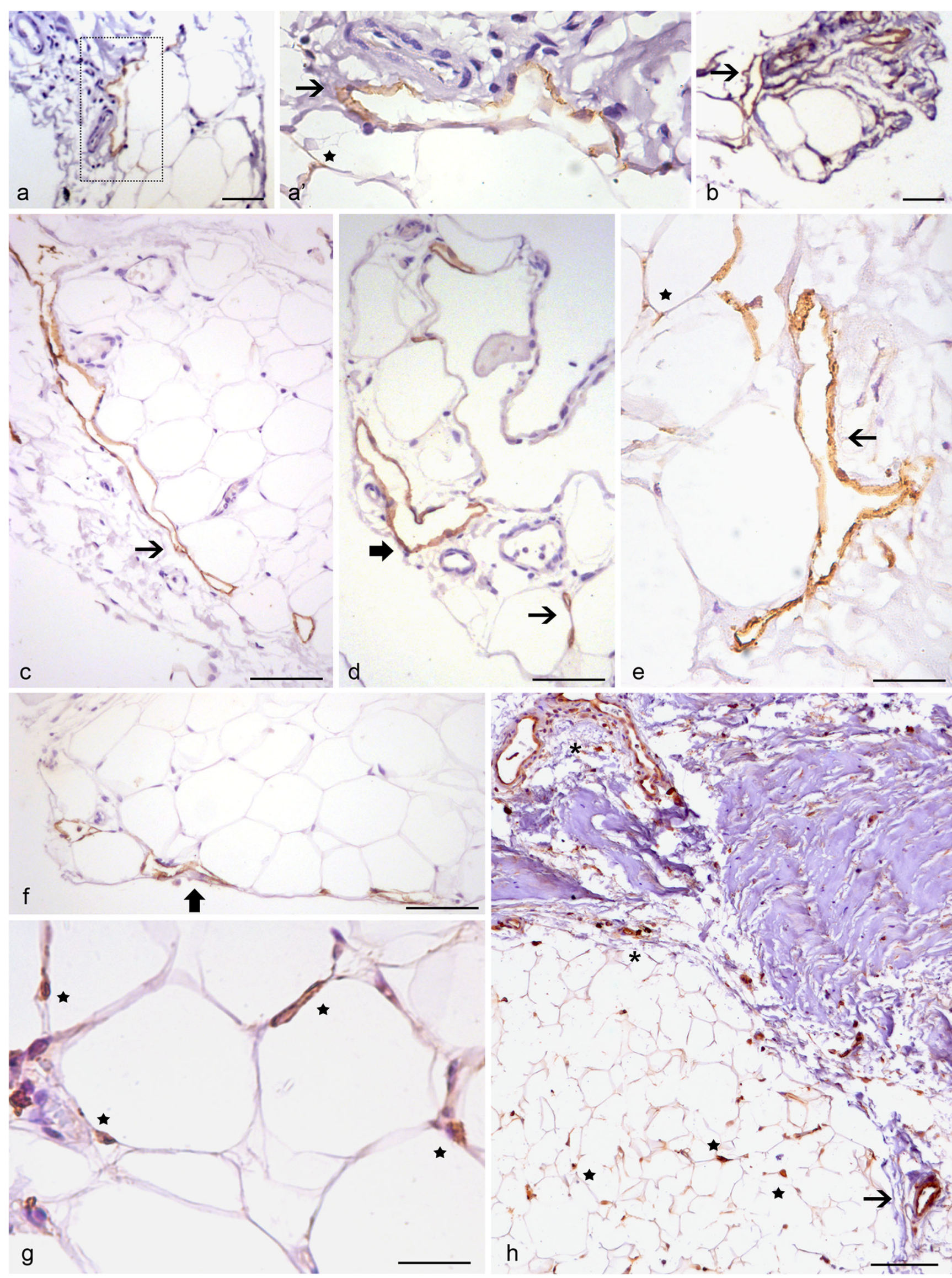

$b^{\circ}$
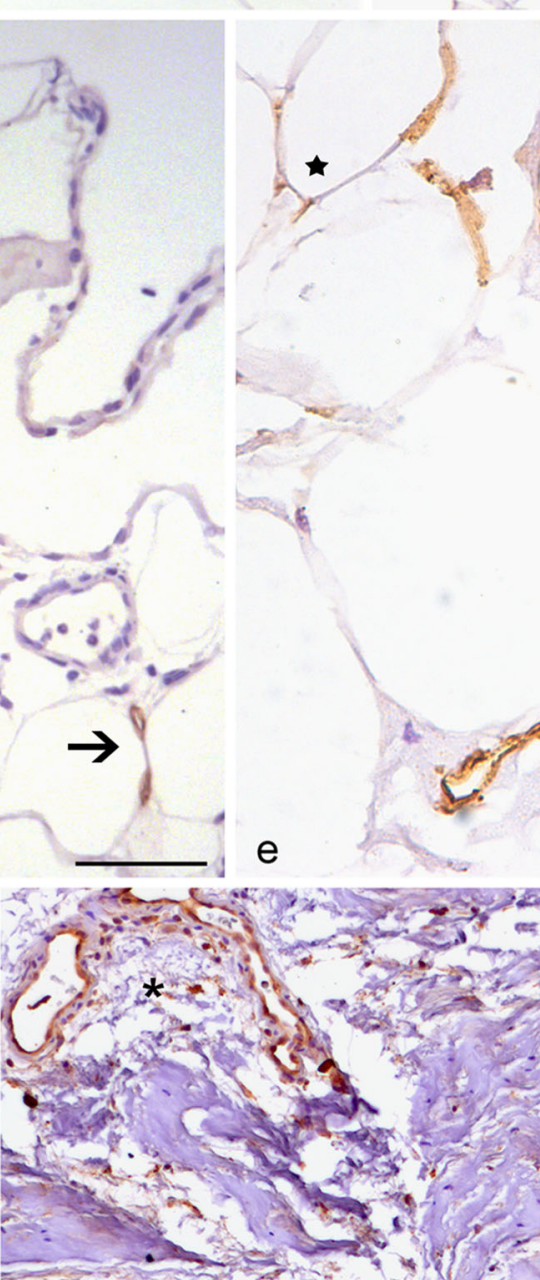

+2 asse?
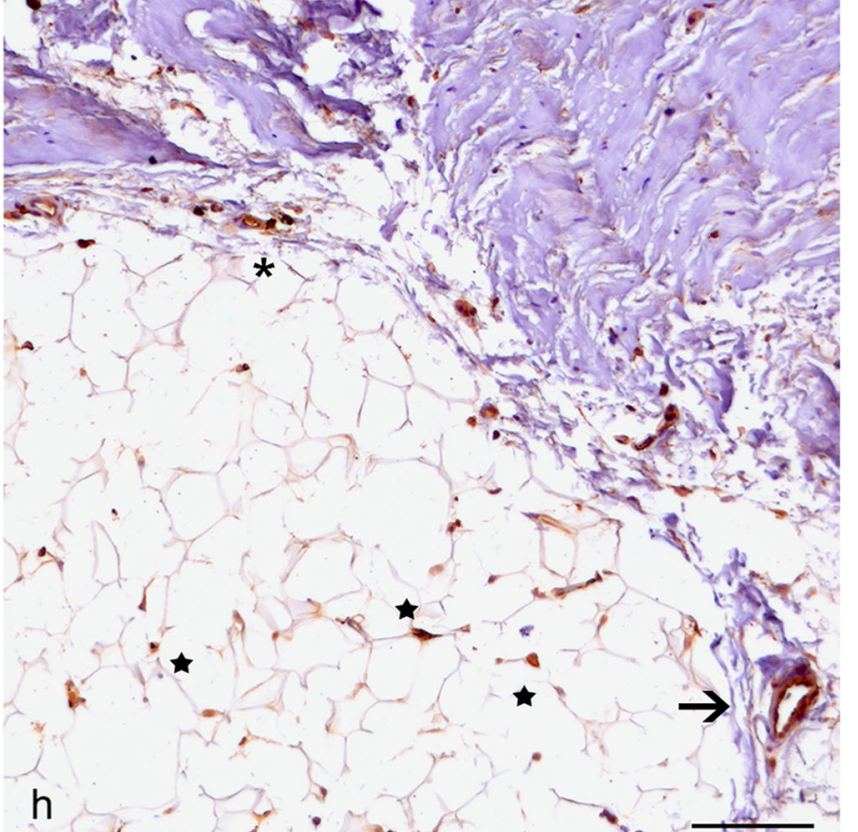
Fig. 1 Immunohistochemical identification of lymphatic vessels in human AT. Endothelial-shaped Lyve1+ (a, b, e) or podoplanin + (c, d, f) cells in close vicinity of adipocytes, perfusing the VAT parenchyma (arrows). (a') Higher magnification of the image shown in a. (d) Structure presenting lymphatic capillary morphology in VAT (arrow). (d, f) Lymphatic vessels in VAT border areas (thick arrows). (h) Lyve1+ vessels in the dermis/hypodermis interface (asterisk) and interlobular fibrous septa (arrow) of SAT. Fusiform Lyve1+ cells scattered in SAT $(\mathrm{g}, \mathrm{h})$ and VAT $\left(\mathrm{a}^{\prime}, \mathrm{e}\right)$ parenchyma (stars). Bars = $100 \mu \mathrm{m}(\mathrm{a}, \mathrm{b}, \mathrm{h}) ; 50 \mu \mathrm{m}(\mathrm{c}-\mathrm{g})$

the adipose subcutaneous tissue but only in its borders (Ryan 1989, 1995).

In mammals, white adipose tissue is divided into subcutaneous adipose tissue (SAT), mainly localised in the gluteus, thighs and abdomen and visceral adipose tissue (VAT), also known as intra-abdominal fat, mostly composed of mesenteric and omental fat. In healthy subjects, the SAT deposits correspond to $80 \%$ of total fat mass, whilst the VAT deposits correspond to 10-20\% (Oka et al. 2010). The VAT compared with the SAT is more cellular, innervated and vascularised (Gealekman et al. 2011; Villaret et al. 2010); contains more leukocytes; and is also more metabolically active, having distinct adipokine secretion profiles (Ibrahim 2010). These divergences might be, at least in part, explained by different embryonic origins, as suggested by recent findings (Chau et al. 2014). Furthermore, an increase in VAT relative to SAT, also called visceral obesity, is a risk factor for the development of cardiovascular diseases, type 2 diabetes, insulin resistance, inflammatory diseases and metabolic syndrome in obese patients (Bergman et al. 2007; Matsuzawa et al. 2011).

The lack of adequate lymphatic drainage in lymphedema is considered one of the main causes of SAT expansion observed in the affected areas (Warren et al. 2007). Additionally, it was observed that a high-fat diet induces lymphatic dysfunction accompanied by capillary dilation in the dermis and reduction of the contractile function of collecting vessels (Aschen et al. 2012; Blum et al. 2014; Sawane et al. 2013; Weitman et al. 2013). Despite these elusive connections between adipose tissue and lymphatic vasculature and the functional requirement of efficient interactions between them, the SAT and VAT lymphatic vascularisation remains incompletely described in humans.

In this study, we investigate lymph vessels in the human SAT and VAT, showing for the first time the lymphatic microvascular structure in human VAT.

\section{Materials and methods}

\section{Patients and samples}

Tissue samples were collected from non-obese patients (14 females and 1 male; mean age $46.3 \pm 2.4$ years) submitted to surgery for hernia corrections in the abdominal region, performed at the Clementino Fraga Filho University Hospital from the Federal University of Rio de Janeiro, after obtaining informed consent. This study was approved by the Clementino Fraga Filho University Hospital Ethics Committee (number 36864514.8.0000.5257). Fragments measuring approximately $125 \mathrm{~mm}^{3}$ were obtained from abdominal SAT and omental VAT. Visceral adipose tissue samples were only collected when surgeries involved access to visceral fat. Exclusion criteria for this study were a body mass index $>30$ and the presence of clinically relevant conditions such as cancer or hepatic, neurological, cardiovascular, infectious and inflammatory diseases.

\section{Antibodies and dyes}

Antibodies used for lymphatic vessel characterisation by immunohistochemistry and immunofluorescence were lymphatic endothelial cell (LEC) markers such as VEGFR3 (1:100, mouse IgG, Millipore; 1:100, goat IgG, R\&D Systems), Lyve1 (1:100, rabbit IgG, Novus Biologicals; 1:100, rabbit IgG R\&D Systems), podoplanin (1:100 or 1:50, mouse IgG, Dako) and Prox1 (1:100, rabbit IgG, Novus Biologicals); CD31 (1:200, mouse IgG, Dako) for blood vessels; the pericyte marker CD146 (1:100, rabbit IgG, Abcam); the pan-leukocyte marker CD45 (1:200, mouse IgG, Abcam) for the identification of haematopoietic cells; and the macrophage marker CD68 (1:50, mouse IgG, Abcam). Secondary goat-antimouse and goat-anti-rabbit antibodies conjugated to Alexa488 or Cy3 (1:1000, Jackson ImmunoResearch), donkey-anti-mouse, donkey-anti-rabbit and donkey-antigoat conjugated to Alexa488 or Cy3 (1:1000, Invitrogen) were used in appropriate combinations. Bodipy 493/503 (1:200, Invitrogen, USA) was used for adipocyte staining. To-Pro-3 (1:1000, Molecular Probes, USA) or DAPI (2.7 mg/ml, Sigma-Aldrich, USA) was used for nucleus labelling.

\section{Immunohistochemistry}

Samples from 9 (SAT, $n=9$; VAT, $n=7$ ) out of the 15 patients used in this study were fixed for $48 \mathrm{~h}$ in buffered formalin and subsequently processed for routine paraffin inclusion. Briefly, sections of $5 \mu \mathrm{m}$ were collected on sylanised sides (Sakura, Netherlands) and used for immunohistochemistry. Following removal of paraffin and rehydration, slides were treated with $50 \mathrm{mM}$ ammonium chloride for $15 \mathrm{~min}$ (Ramos-Vara 2005), permeabilised with PBS-Triton $0.5 \%$ and incubated for 15 min with $30 \%$ hydrogen peroxide. Heated antigen retrieval was performed with citrate buffer $(\mathrm{pH}$ 6.0) for 15 min (Pusztaszeri et al. 2006), followed by PBS washing and 60-min incubation in blocking buffer containing $5 \%$ of BSA and/or $5 \%$ of normal goat or 


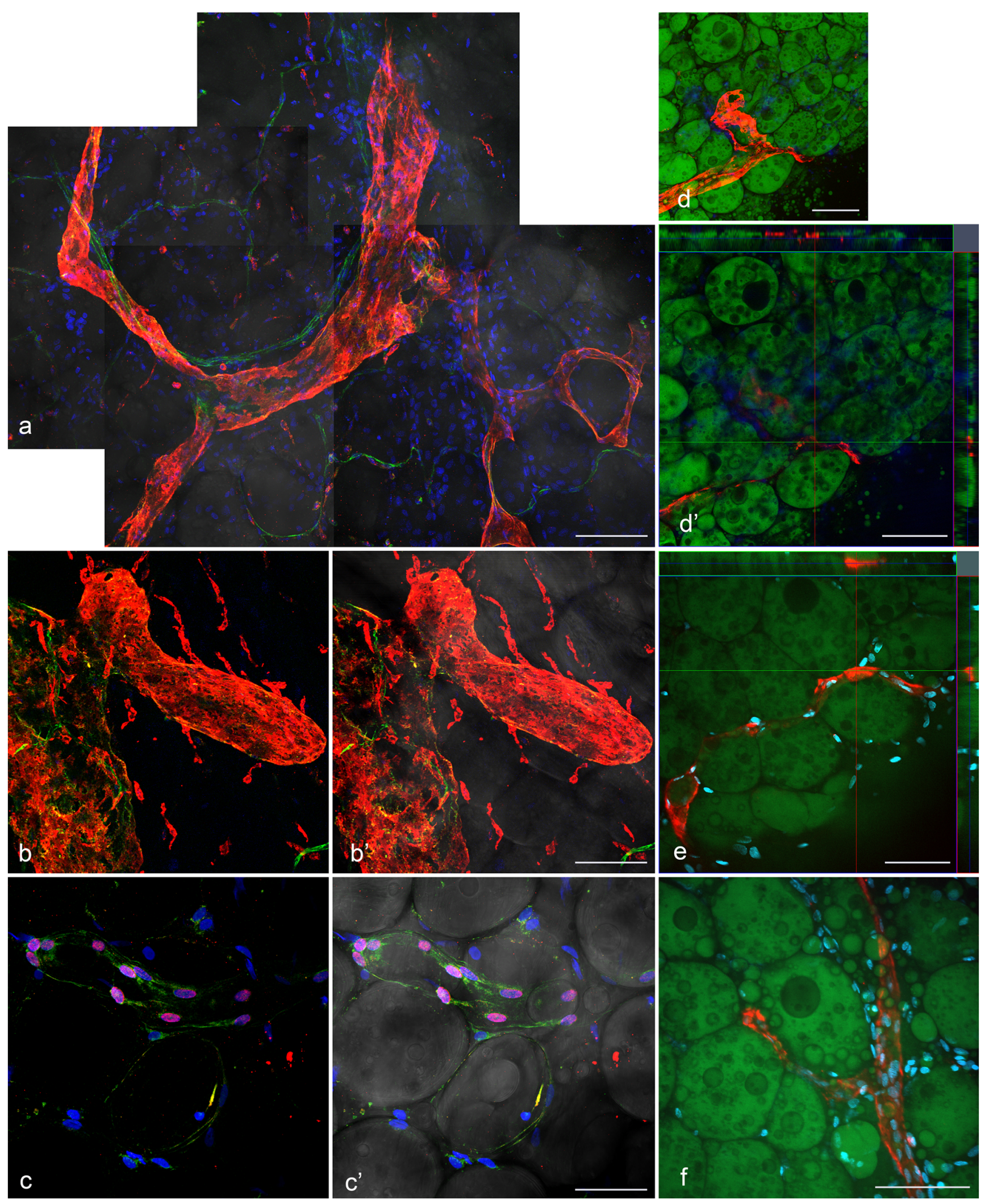

donkey serum, before overnight incubation with primary antibodies. LSAB kit (Dako, USA) was used for secondary labelling and $\mathrm{DAB}$ for chromogenic revelation.
At least 2 sections per patient/stain were entirely observed under a microscope. Slides were counterstained with Harris haematoxylin, mounted with Entellan 
Fig. 2 Projections of 3D reconstructions of the lymphatic vasculature in human VAT. (a) Lyve1+ lymphatic and CD31+ blood vessels (red and green, respectively) presenting varied calibres within the tissue. Note the proximity between blood vessel and higher calibre lymphatic vessels. (b) Lyve1+CD31+ (Lyve, red; CD31, green) or (c) VEGFR3+Prox1+ (VEGFR3, green; Prox1, red) initial lymphatic vessels presenting typical morphology. (d, e) Lyve1+ lymphatic vessel permeating adipocytes stained with Bodipy (red and green, respectively). (d') Orthogonal slices from the image shown in $\mathrm{d}$ and from one additional example (e) of Lyve1+ vessels located in between adipocytes within the VAT parenchyma, as observed in lateral and superior boxes. (f) Lyve1+ initial vessel draining to a higher calibre vessel surrounded by juxtaposed adipocytes stained with Bodipy. Bright field images are merged with fluorescence images in $\mathrm{a}, \mathrm{b}^{\prime}$ and $\mathrm{c}^{\prime}$. Nuclei were stained with To-Pro or DAPI (blue). Bars $=100 \mu \mathrm{m}(\mathrm{a}, \mathrm{b}, \mathrm{d}-\mathrm{f})$ and $50 \mu \mathrm{m}$ (c)

(Merck-Millipore, USA) and photographed with an Eclipse E400 microscope (Nikon, Japan) equipped with QCapture software (SpectraServices, USA) or, with a Zeiss Axiovert 200M microscope (Carl Zeiss, Germany) equipped with Axiocam HRc and ZEN software (Carl Zeiss, Germany).

\section{Whole mount staining}

Tissue samples from 11 (SAT, $n=11$; VAT, $n=7$ ) out of the 15 patients used in this study were fixed in $4 \%$ freshly prepared paraformaldehyde for $2 \mathrm{~h}$ immediately after surgical resection. Whole mount processing protocol was performed as previously described (Xue et al. 2010; Daquinag et al. 2013). A minimum of 3 fragments measuring approximately $9 \mathrm{~mm}^{3}$ were separated, washed in PBS-Triton $0.1 \%$ and incubated with $5 \%$ goat or donkey serum for $1 \mathrm{~h}$. Samples were incubated for $48 \mathrm{~h}$ at 4 ${ }^{\circ} \mathrm{C}$ with primary antibodies under rotation, followed by PBS-Triton $0.1 \%$ washing and incubation with secondary antibodies conjugated to fluorochromes and the nuclear marker To-Pro-3 or DAPI. Bodipy staining (1:200) was performed under rotation for $30 \mathrm{~min}$. After washing with PBS, fragments were mounted with Vectashield (Vector Laboratories, UK) and sealed with coverslips. Optical slice images were obtained serially using a Zeiss LSM510 Meta laser scanning confocal microscope (Carl Zeiss, Germany) covering 30 to $300 \mu \mathrm{m}$ of thickness in the $z$ axis, or with an Axio Observer.Z1 inverted microscope equipped with a CSU-X1A 5000 Yokogawa Spinning Disk confocal unit (Carl Zeiss, Germany) with an EMCCD Camera QImaging Rolera em-c2 (Teledyne Technologies, Netherlands) run by Zen 2011 software (Carl Zeiss Microscopy, Germany). Zen software was used to process z-stacked images and for $3 \mathrm{D}$ reconstruction. Photoshop CC 2013 (Adobe, USA) was used to merge the fields. At least 3 fragments measuring approximately $9 \mathrm{~mm}^{3}$ were entirely observed in all the focal plans with $\mathrm{a} \times 10$ objective. Lymphatic vessel counting was performed in 5 fields of z-stack projections per patient stained with Lyve1 or VEGFR3. Each branch of the vessels was counted as one. Statistical analysis was performed using GraphPad Prism 5.0 (GraphPad Software, USA). An unpaired two-tailed $t$ test was used to compare both groups.

\section{Results}

\section{Lymphatic vessels are abundant in human VAT but are rare in SAT}

Immunohistochemistry performed in samples from 9 patients (SAT, $n=9$; VAT, $n=7$ ) revealed the presence of small- and medium-calibre vessels lined by Lyve1+ or podoplanin cells in the borders and within the lobes of VAT samples in all 7 patients (Fig. 1a-f). In the SAT samples, lymphatic vessels were only detected in 2 out of 9 patients. When observed, they were found in the subcutaneous vascular plexus or in the fibrous septa crossing the fat pads (Fig. 1h). In both VAT and SAT, isolated Lyve1+ cells were observed in close contact with adipocytes, without forming vascular structures (Fig. 1a', e, g, h).

Lymphatic vessel distribution was investigated more thoroughly by the whole mount technique (Xue et al. 2010; Daquinag et al. 2013) using confocal microscopy and $3 \mathrm{D}$ reconstruction. In the VAT, Lyve1+ lymphatic vessels with different calibres were observed (Fig. 2). Wider vessels juxtaposed to CD31+ blood vessels were identified (Fig. 2a). CD31+ blood capillaries were detected in close proximity with adipocytes, as previously described (Gao et al. 2015). Lymphatic capillaries characterised by the detection of Lyve1 (Fig. 2b) or by the detection of VEGFR3 and the LEC nuclear marker Prox1 (Fig. 2c) exhibited classical morphology, presenting blunt ends and apparent loose intercellular spaces (Baluk et al. 2007). Their distribution pattern was distinct from that of blood capillaries. The lymphatic vessels were observed in between unilocular adipocytes, within the VAT parenchyma (Fig. 2d-f).

As opposed to the VAT, where Lyve1+ or VEGFR3+ vessels were readily observed in all 7 patients (7/7), in the SAT, albeit extensive and arduous observation of samples from 11 patients, Lyve1+ or VEGFR3+ lymphatic vessels were only found in 3 of them (3/11). Importantly, lymphatic vessels were detected in the adjacent dermis of all patients (Fig. $3 \mathrm{a}, \mathrm{d}$ ), nevertheless, when the interface between the dermis and adipose tissue was analysed, there was no continuity of the vessels to the SAT (Fig. 3b, e). The Lyve1+ (Fig. 3c) or VEGFR3+ (Fig. 3f) vessels were found predominantly in the fibrous connective interlobular tissue within the adipose tissue, presenting a diameter 

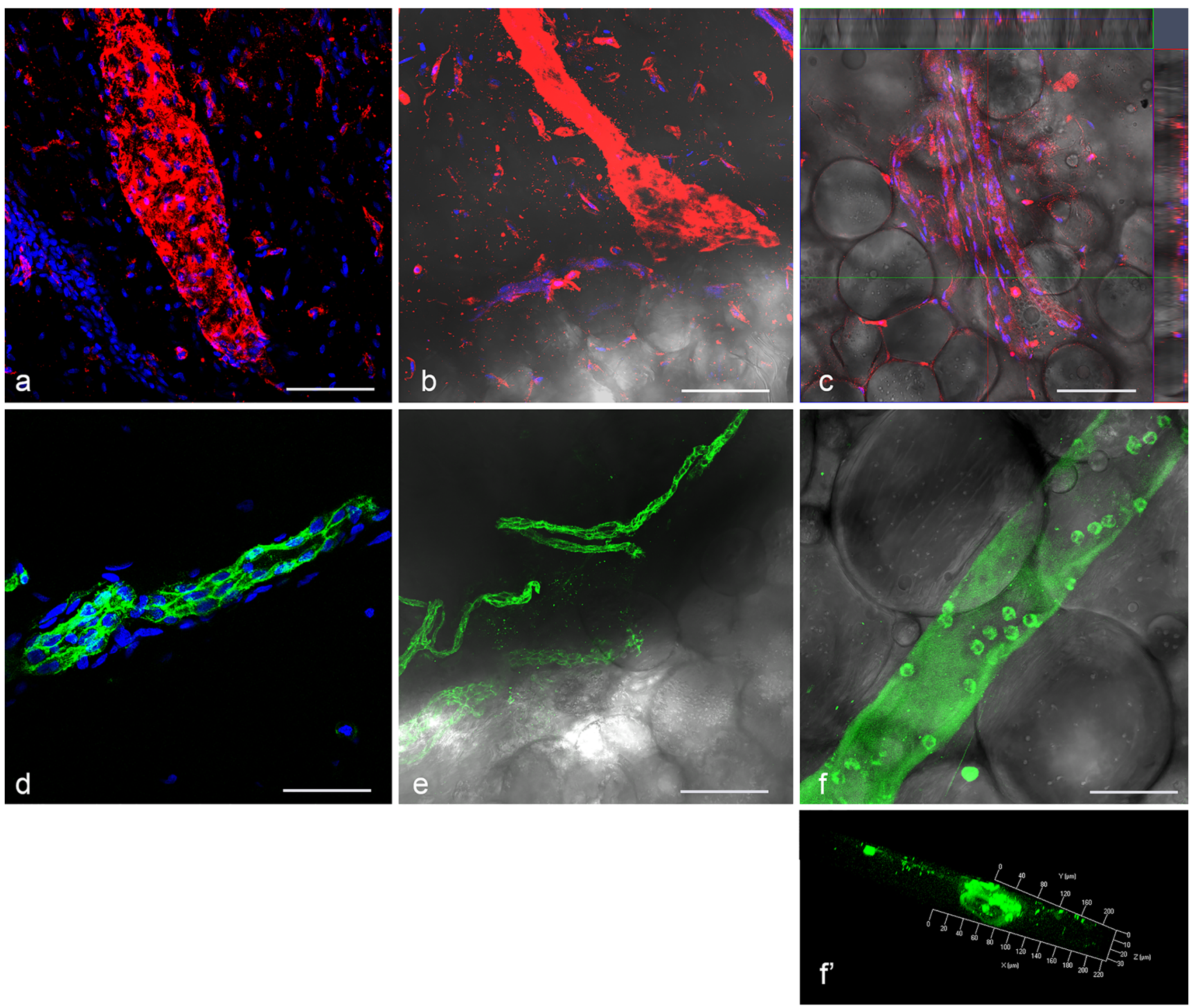

Fig. 3 Projections of 3D reconstructions of the lymphatic vasculature in human SAT. Lyve1+ (a, red) and VEGFR3 + (d, green) lymphatic vessels in the dermis presenting typical morphology. Lyve1+ (b, red) and VEGFR3+ (e, green) in adjacent dermis showing no continuity to SAT in the hypodermis. (c) Lyve1+ cells forming a vascular continuum in fibrous regions of interlobular space. Orthogonal slices in lateral and superior boxes show the presence of vessels in fibrous regions over the

of 30-50 $\mu \mathrm{m}$ (Fig. 3f, $\mathrm{f}^{\prime}$ ). Additionally, round VEGFR3+ cells were associated with lymphatic vessels. These were clearly distinguished from the LECs by their morphology, localisation and labelling intensity (Fig. 3f). A basic scoring method to quantify differences observed between SAT and VAT vessel numbers was used. Individual branches of Lyve1+ or VEGFR3+ lymphatic vessels were scored as 1 vessel. Whilst the mean vessel count in the SAT group was $2.727( \pm 1.526)$, the VAT group showed a 4.96 times higher vessel count $(13.43 \pm 2.202)$, significantly different from the amount found in $\operatorname{SAT}(p=0.0008)$ (Fig. 4). adipocytes. (f) VEGFR3+ vessels in fibrous regions revealed by the vessel alignment with shadow and refractional areas in the bright field images. ( $\left.f^{\prime}\right)$ Perspective projection of the vessel shown in image $f$ exhibiting a 30-50- $\mathrm{m}$ lumen with bright VEGFR3+ round cells in its interior. Bright field images are merged with fluorescence images in b, c, $\mathrm{e}$ and $\mathrm{f}$. Nuclei were stained with To-Pro (blue) Bars $=100 \mu \mathrm{m}(\mathrm{a}-\mathrm{c}, \mathrm{e})$; $50 \mu \mathrm{m}(\mathrm{d}, \mathrm{f})$

\section{Numerous Lyve1+ cells are observed in association with blood vessels from the adipose tissue}

Three-dimensional reconstructions and z-stack projections showed Lyve1+ cells surrounding adipocytes and neighbouring adipocyte-associated CD31+ capillaries. These cells were forming neither a lymphatic continuum nor vascular structures (Fig. 5a, b). Aiming to address whether these cells were pericytes, as their morphology was compatible with that of pericytes associated to capillaries, double labelling with CD146 was performed. Most of the cells were Lyve1+CD146-, 


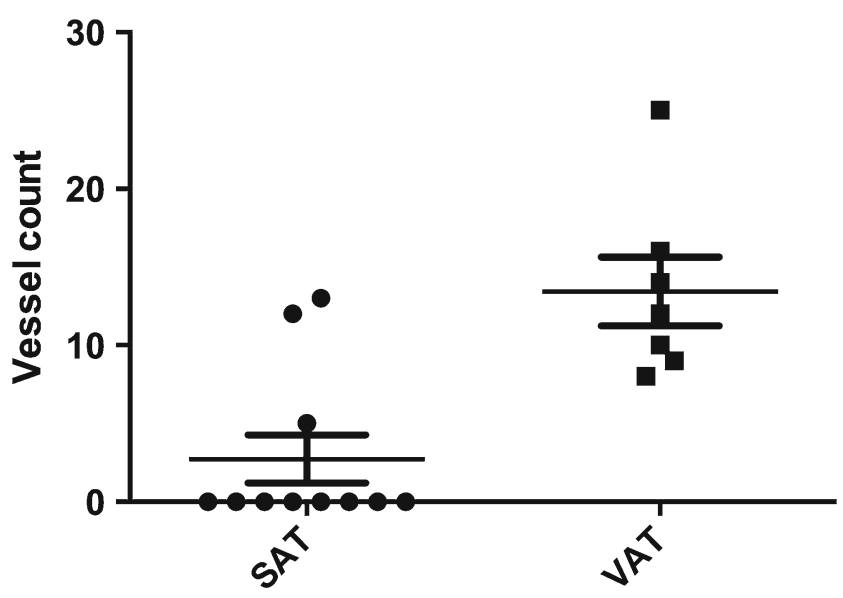

Fig. 4 Morphometric analysis of lymphatic vessels in SAT and VAT. Individual vessel branches were scored as one vessel in 5 fields of zstack projections per patient stained with Lyve1 or VEGFR3. Data points are individual patients. Bars are group means \pm SEM. Statistical analysis between both groups was performed to obtain the $p$ value using an unpaired two-tailed $t$ test. $p=0.0008$

although Lyve1+CD146+ cells could also be observed (Fig. 5c). Round Lyve1+ cells allusive to haematopoietic cells were also detected (Fig. 5c'), similarly to VEGFR3+ cells (Fig. 3f). Double staining with CD45 confirmed their haematopoietic origin and showed Lyve1+CD45+ fusiform cells permeating the tissue, suggesting morphologically and phenotypically that these could be macrophages (Fig. 5d). The macrophage phenotype was verified using double staining with the bona fide marker CD68. Although Lyve1+CD68- and Lyve1-CD68+ could be observed, most of the scattered Lyve1+ cells were also CD68+ in both SAT and VAT (Fig. 5e, f).

\section{Discussion}

In the present study, we could not identify initial lymphatic capillaries within the human SAT. However, lymph vessels were clearly observed in the interlobular fibrous septa, in the fibrous septa crossing the adipose parenchyma, as well as in association with the subcutaneous vascular plexus. These results are in accordance with morphological studies from Ryan (1989, 1995), who described lymphatic vessels only in the SAT borders, as well as with updated studies on dermal microcirculation, in which lymphatic capillaries remain mostly unreported (Breslin et al. 2019), despite eventual imaging of collecting lymphatics in the SAT (Tashiro et al. 2017). Regardless of extensive analysis of samples using immunohistochemistry and/or whole mount, we could not identify a continuity of Lyve1+ or VEGFR3+ lymph vessels from the dermis into the adjacent adipose tissue parenchyma. Lymph vessels usually collapse in tissue sections due to their delicate structure, which hampers their visualisation (Jackson 2003). Additionally, the inherent technical difficulties from adipose tissue preparation could impair proper microscopic observation of its structural details (Xue et al. 2010). However, it is unlikely that the lymphatic capillaries are abundant in the SAT but undetected due to methodological issues, since their observation in the VAT samples was possible. Furthermore, previous studies based on colloid technetium injection in fat pads indicated poor lymphatic drainage in the SAT parenchyma, showing slow or absent depuration (Mortimer et al. 1990; Ryan 1989) even after vigorous massage (Lubach et al. 1996; Ryan 1997; Ryan and De Berker 1995). Hence, taken together with the literature, our data indicate that human SAT, in contrast to most vascularised human tissues, including VAT, does not present a lymphatic capillary network intimately surrounding adipocytes, accompanying the blood capillaries, as could be expected.

It should be noted though, that the dense blood capillary network in SAT lobes suggests lymph formation in the interstitial space (Ikomi et al. 2012; Scallan and Huxley 2010; Von der Weid and Rainey 2010) and that many studies have shown increased local lymphatic flow after skin massaging (Ikomi and SchmidSchonbein 1995; Ikomi and Schmid-Schönbein 1996; Lubach et al. 1996; Ryan and De Berker 1995). For this reason, the possibility of SAT interstitial fluid drainage via lymphatics from the dermis/hypodermis interface, from the interlobar septa or by mechanisms that are independent of classical initial vessels in proximity to blood capillaries should not be discarded. Further investigations on SAT lymphatic drainage and flow are needed to clarify these questions.

Conversely to the observations in SAT, blunt-ended capillaries converging to higher calibre lymphatic vessels presenting classical morphology (Oliver and Alitalo 2005; Schulte-Merker et al. 2011) were observed in human VAT. Vessels were detected surrounding fat lobes, in the fibrous septa, as well as perfusing the tissue parenchyma. To our knowledge, this is the first demonstration of lymphatic capillaries and vessels in human VAT using specific LEC molecular markers.

In the present study, we also showed fibroblastoid Lyve1+ cells in between adipocytes and with perivascular localisation. Although occasionally expressing the pericyte maker CD146, Lyve1+CD45+ cells with such morphology were abundant, indicating a haematopoietic phenotype. Round-shaped Lyve1+CD45+ and VEGFR3+ CD45+ cells were also detected. Most of the fusiform Lyve1+ cells scattered in between adipocytes were also CD68+, confirming the macrophage phenotype, in accordance with studies reporting Lyve1+ or VEGFR3+ macrophages based on their morphology and on the lack of a lymphatic continuum formation (Haiko et al. 2008; Martinez-Santibañez et al. 2014; Sawane et al. 2013; Wang et al. 2014).

The SAT and VAT lymphatic architectural particularities described in the present work open a new front of 
Fig. 5 Abundant perivascular Lyve1+ cells in human AT. Threedimensional reconstruction projections (a, $\mathrm{c}-\mathrm{f}$ ) and perspective (b) of SAT (a-e) and VAT (f) samples labelled with Lyve1. (a) Blood capillaries CD31+ (green) Lyve1- (red) in intimate proximity with adipocytes. Fusiform Lyve1+ cells with perivascular localisation or surrounding adipocytes. (b) Perspective projection evidencing sparse Lyve 1+ cells lacking the formation of a lymphatic vascular continuum. (c) CD146+ fusiform cells (c, green), Lyve 1+ round and fusiform cells (c', red), Lyve1+ CD146+ cells ( $\mathrm{c}^{\prime \prime}$, arrows) and Lyve1+CD146- cells (c", asterisk). (d) CD45+ round and fusiform cells (d, green), Lyve1+ round and fusiform cells ( $\mathrm{d}^{\prime}$, red), Lyve $1+C D 45+$ cells (d", arrows) and Lyve1+CD45- cells (d", asterisk). (e) CD68+ fusiform cells (e, green), Lyve1+ fusiform cells (e', red), Lyve1+CD68+ cells (e", arrows) and Lyve1 -CD68+ cells (e", asterisk) in the SAT. (f) CD68+ round and fusiform cells (f, green), Lyve1+ round and fusiform cells ( $\mathrm{f}^{\prime}$, red), Lyve1+CD68+ fusiform cells ( $\mathrm{f}^{\prime \prime}$, arrows), Lyve1+CD68- cells (f", asterisk) and Lyve1-CD68+ cells $\left(\mathrm{f}^{\prime \prime}\right.$, star). Bright field images are merged with fluorescence images in $\mathrm{e}^{\prime \prime}$ and $\mathrm{f}^{\prime \prime}$. Nuclei were stained with To-Pro or DAPI (blue).

Images are representative of SAT and VAT samples. Bars $=100 \mu \mathrm{m}$
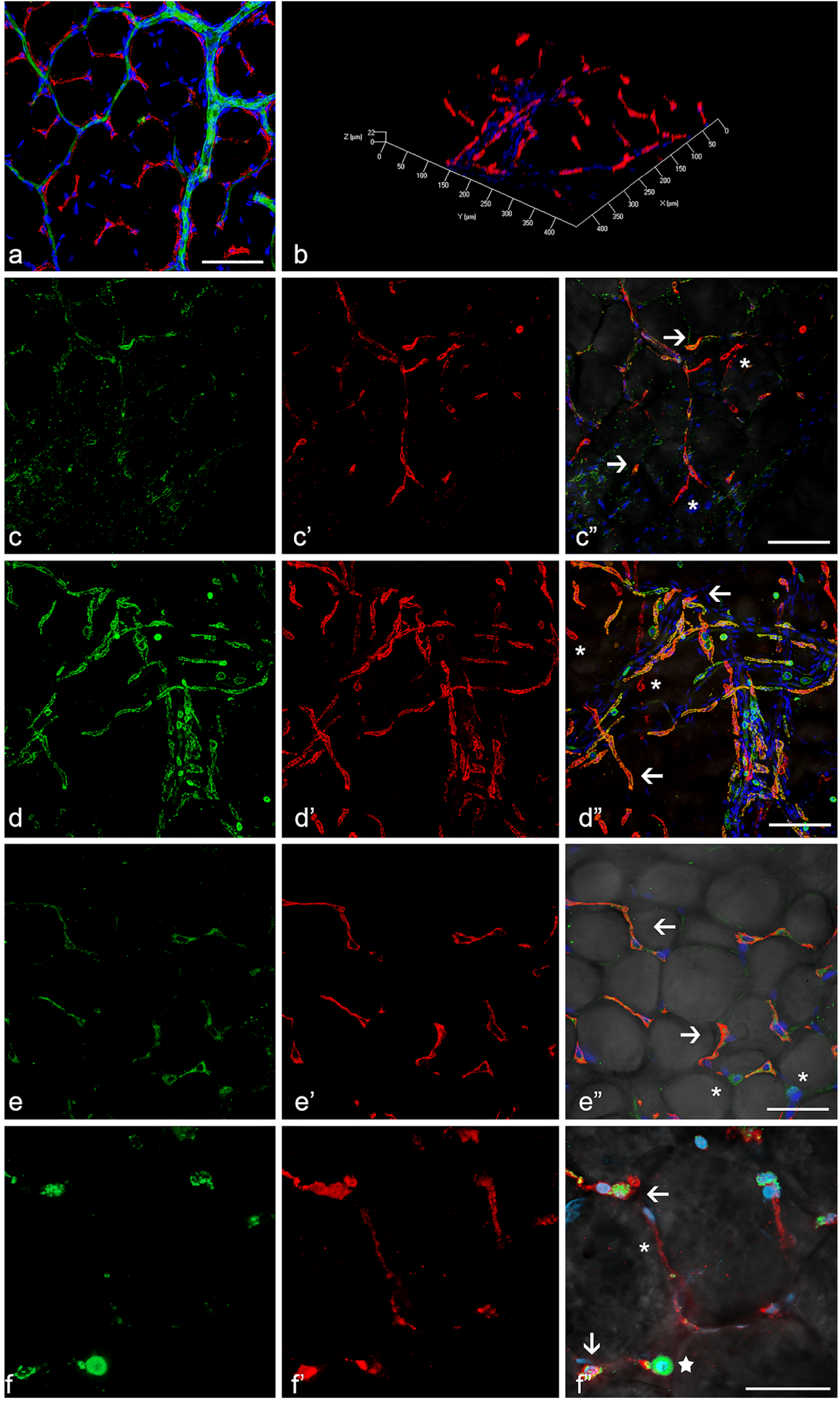

investigation in adipose tissue biology, with important implications in the understanding of lymphedema, as well as in elucidating the different roles of these depots in obesity, which is under investigation in our group. 
Acknowledgements The authors would like to thank Dr. Mikhail Kolonin, for the suggestions and for the whole mount protocol; Mateus N Freitas and Luzia F G Caputo, for the technical support with microscopy and histotechnology facilities at Fiocruz; Plataforma de Microscopia Óptica de Luz Gustavo de Oliveira Castro (PLAMOL), a light microscopy facility of Instituto de Biofísica Carlos Chagas Filho - UFRJ, where part of the imaging was performed.

Funding information This work was partly funded by the Brazilian National Counsel of Technological and Scientific Development and by The Royal Free Charity (grant no. 536995).

Compliance with ethical standards All procedures performed in studies involving human participants were in accordance with the ethical standards of the institutional and/or national research committee and with the 1964 Helsinki declaration and its later amendments or comparable ethical standards.

Open Access This article is distributed under the terms of the Creative Commons Attribution 4.0 International License (http:// creativecommons.org/licenses/by/4.0/), which permits unrestricted use, distribution, and reproduction in any medium, provided you give appropriate credit to the original author(s) and the source, provide a link to the Creative Commons license, and indicate if changes were made.

\section{References}

Aschen S, Zampell JC, Elhadad S, Weitman E, De Brot M, Mehrara BJ (2012) Regulation of adipogenesis by lymphatic fluid stasis: part II. Expression of adipose differentiation genes. Plast Reconstr Surg 129:838-847

Baluk P, Fuxe J, Hashizume H, Romano T, Lashnits E, Butz S, Vestweber D, Corada M, Molendini C, Dejana E, McDonald DM (2007) Functionally specialized junctions between endothelial cells of lymphatic vessels. J Exp Med 204:2349-2362

Bergman RN, Kim SP, Hsu IR (2007) Abdominal obesity: role in the pathophysiology of metabolic disease and cardiovascular risk. Am J Med 120:S3-S8

Blum KS, Karaman S, Proulx ST, Ochsenbein AM, Luciani P, Leroux JC, Wolfrum C, Detmar M (2014) Chronic high-fat diet impairs collecting lymphatic vessel function in mice. PLoS One 9:e94713

Breslin JW, Yang Y, Scallan JP, Sweat RS, Adderley SP, Murfee WL (2019) Lymphatic vessel network structure and physiology. In Comprehensive physiology, DM Pollock (Ed). Compr Physiol 9:207-299

Chau YY, Bandiera R, Serrels A, Martínez-Estrada OM, Qing W, Lee M, Hastie N (2014) Visceral and subcutaneous fat have different origins and evidence supports a mesothelial source. Nat Cell Biol 16:367-375

Daquinag AC, Souza GR, Kolonin MG (2013) Adipose tissue engineering in three-dimensional levitation tissue culture system based on magnetic nanoparticles. Tissue Eng Part C Methods 19:336-344

Gao Z, Zhang J, Henagan TM, Lee JH, Ye X, Wang H, Ye J (2015) P65 inactivation in adipocytes and macrophages attenuates adipose inflammatory response in lean but not in obese mice. Am J Physiol Endocrinol Metab 308:E496-E505

Gealekman O, Guseva N, Hartigan C (2011) Depot-specific differences and insufficient subcutaneous adipose tissue angiogenesis in human obesity. Circulation 123:186-194

Haiko P, Makinen T, Keskitalo S, Taipale J, Karkkainen MJ, Baldwin ME, Stacker SA, Achen MG, Alitalo K (2008) Deletion of vascular endothelial growth factor C (VEGF-C) and VEGF-D is not equivalent to VEGF receptor 3 deletion in mouse embryos. Mol Cell Biol $28: 4843-4850$
Ibrahim MM (2010) Subcutaneous and visceral adipose tissue: structural and functional differences. Obes Rev 11:11-18

Ikomi F, Schmid-Schonbein GW (1995) Lymph transport in the skin. Clin Dermatol 13:419-427

Ikomi F, Schmid-Schönbein GW (1996) Lymph pump mechanics in the rabbit hind leg. Am J Phys 271:H173-H183

Ikomi F, Kawai Y, Ohhashi T (2012) Recent advance in lymph dynamic analysis in lymphatics and lymph nodes. Ann Vasc Dis 5:258-268

Jackson DG (2003) The lymphatics revisited: new perspectives from the hyaluronan receptor LYVE-1. Trends Cardiovasc Med 13:1-7

Jeltsch M, Tammela T, Alitalo K, Wilting J (2003) Genesis and pathogenesis of lymphatic vessels. Cell Tissue Res 314:69-84

Kohan AB, Yoder SM, Tso P (2011) Using the lymphatics to study nutrient absorption and the secretion of gastrointestinal hormones. Physiol Behav 105:82-88

Lubach D, Lüdemann W, Berens von Rautenfeld D (1996) Recent findings on the angioarchitecture of the lymph vessel system of human skin. Br J Dermatol 135:733-737

Martel C, Li W, Fulp B, Platt AM, Gautier EL, Westerterp M, Randolph GJ (2013) Lymphatic vasculature mediates macrophage reverse cholesterol transport in mice. J Clin Invest 123:1571-1579

Martinez-Santibañez G, Cho KW, Lumeng CN (2014) Imaging white adipose tissue with confocal microscopy. Methods Enzymol 537:17-30

Matsuzawa Y, Funahashi T, Nakamura T (2011) The concept of metabolic syndrome: contribution of visceral fat accumulation and its molecular mechanism. J Atheroscler Thromb 18(8):629-639

Montani JP, Carroll JF, Dwyer TM, Antic V, Yang Z, Dulloo G (2004) Ectopic fat storage in heart, blood vessels and kidneys in the pathogenesis of cardiovascular diseases. Int J Obes Relat Metab Disord 28:S58-S65

Mortimer PS, Simmonds R, Rezvani M, Robbins M, Hopewell JW, Ryan TJ (1990) The measurement of skin lymph flow by isotope clearance-rehability, reproducibility, injection dynamics, and the effect of massage. J Invest Derm 95:677-682

Nanjee MN, Cooke CJ, Wong JS, Hamilton RL, Olszewski WL, Miller NE (2001) Composition and ultrastructure of size subclasses of normal human peripheral lymph lipoproteins: quantification of cholesterol uptake by HDL in tissue fluids. J Lipid Res 42:639-648

Oka R, Miura K, Sakurai M, Nakamura K, Yagi K, Miyamoto S, Moriuchi T, Mabuchi H, Koizumi J, Nomura H, Takeda Y, Inazu A, Nohara A, Kawashiri M, Nagasawa S, Kobayashi J, Yamagishi M (2010) Impacts of Visceral Adipose Tissue and Subcutaneous Adipose Tissue on Metabolic Risk Factors in Middle-aged Japanese. Obesity, 18: 153-160

Oliver G, Alitalo K (2005) The lymphatic vasculature: recent progress and paradigms. Annu Rev Cell Dev Biol 21:457-483

Pusztaszeri MP, Seelentag W, Bosman FT (2006) Immunohistochemical expression of endothelial markers CD31, CD34, von Willebrand factor, and Fli-1 in normal human tissues. J Histochem Cytochem 54(4):385-395

Ramos-Vara JA (2005) Technical aspects of immunohistochemistry. Vet Pathol 42(4):405-426

Randolph GJ, Miller NE (2014) Lymphatic transport of high-density lipoproteins and chylomicrons. J Clin Investig 124:929-935

Ryan TJ (1989) Structure and function of lymphatics. J Invest Dermatol 93:18S-24S

Ryan TJ (1995) Lymphatics and adipose tissue. Clin Dermatol 13:493-498

Ryan TJ (1997) Landmarks in the understanding of lymphatic function and the management of edema. Clin Dermatol 13:417-418

Ryan TJ, De Berker D (1995) The interstitium, the connective tissue environment of the lymphatic, and angiogenesis in human skin. Clin Dermatol 13:451-458

Sawane M, Kajiya K, Kidoya H, Takagi M, Muramatsu F, Takakura N (2013) Apelin inhibits diet-induced obesity by enhancing lymphatic and blood vessel integrity. Diabetes 62:1970-1980 
Scallan JP, Huxley VH (2010) In vivo determination of collecting lymphatic vessel permeability to albumin: a role for lymphatics in exchange. J Physiol 588:243-254

Schulte-Merker S, Sabine A, Petrova TV (2011) Lymphatic vascular morphogenesis in development, physiology, and disease. J Cell Biol 193:607-618

Tammela T, Alitalo K (2010) Lymphangiogenesis: molecular mechanisms and future promise. Cell 140:460-476

Tashiro K, Feng J, Wu S, Mashiko T, Kanayama K, Narushima M, Uda H, Miyamoto S, Koshima I, Yoshimura K (2017) Pathological changes of adipose tissue in secondary lymphoedema. Br J Dermatol 177:158-167

Villaret A, Galitzky J, Decaunes P, Estève D, Marques MA, Sengenès C, Chiotasso P, Tchkonia T, Lafontan M, Kirkland JL, Bouloumié A (2010) Adipose tissue endothelial cells from obese human subjects: differences among depots in angiogenic, metabolic, and inflammatory gene expression and cellular senescence. Diabetes 59:2755-2763

Von der Weid PY, Rainey KJ (2010) Review article: lymphatic system and associated adipose tissue in the development of inflammatory bowel disease. Aliment Pharmacol Ther 32:697-711
Wang Y, Oliver G (2010) Current views on the function of the lymphatic vasculature in health and disease. Genes Dev 24:2115-2126

Wang XN, McGovern N, Gunawan M, Richardson C, Windebank M, Siah TW, Haniffa M (2014) A three-dimensional atlas of human dermal leukocytes, lymphatics, and blood vessels. J Invest Dermatol 134:965-974

Warren AG, Brorson H, Borud LJ, Slavin SA (2007) Lymphedema: a comprehensive review. Ann Plast Surg 59:464-472

Weitman ES, Aschen SZ, Farias-Eisner G, Albano N, Cuzzone D, Ghanta S, Mehrara BJ (2013) Obesity impairs lymphatic fluid transport and dendritic cell migration to lymph nodes. PLoS One 8:e70703

Xue Y, Lim S, Bråkenhielm E, Cao Y (2010) Adipose angiogenesis: quantitative methods to study microvessel growth, regression and remodeling in vivo. Nat Protoc 5:912-920

Publisher's note Springer Nature remains neutral with regard to jurisdictional claims in published maps and institutional affiliations. 